\title{
NOTE ON THE PERIOD OF A MARK IN A FINITE FIELD
}

\author{
BY MORGAN WARD
}

1. Introduction. If $p$ is a fixed prime, and

$$
F(x)=x^{k}-c_{1} x^{k-1}-\cdots-c_{k},
$$

where $c_{1}, \cdots, c_{k}$ are rational in tegers, is a polynomial which is irreducible modulo $p$, the period of a mark $\alpha$ associated with the polynomial $F(x)$ in the finite field $\mathcal{F}$ of order $p^{k}$ is fundamental not only in the theory of finite fields, ${ }^{*}$ but also in many allied arithmetical investigations involving recurring series. $\dagger$

Our information about the actual value of this period is disappointingly meagre beyond the well known facts that it is a divisor of $p^{k}-1$ and that there actually exist polynomials $F(x)$ for which the period equals $p^{k}-1$. I prove here the following additional result.

ThEOREM. Let $\tau$ denote the period of a mark $\alpha$ associated with the irreducible polynomial $F(x)$ modulo $p$ in the finite field $\mathcal{F}$ of order $p^{k}$, and let $\omega$ be the least positive value of $n$ such that $\alpha^{n}$ is congruent to a rational integer modulo $p$. $\ddagger$ Then $\tau=\delta \theta \omega$, where $\theta$ is the exponent to which norm $\alpha$ belongs modulo $p$, while $\delta$ is an integer dividing the greatest common divisor of $k$ and $p-1$, and multiplying the greatest common divisor of $\theta$ and the integer $\sigma=\left(p^{k}-1\right) /(\omega(p-1))$.

* See, for example, Dickson, Linear Groups, 1901, Chapters 1-3.

$\dagger$ If $\Omega_{n+k}=c_{1} \Omega_{n+k-1}+\cdots+c_{k} \Omega_{n}$ is the difference equation associated with the polynomial $F(x)$, the period of $\alpha$ is the period modulo $p$ of every sequence of rational integers satisfying the difference equation. (See Ward, Transactions of this Society, vol. 35 (1933), pp. 600-628, and the references given there.) The period of $\alpha$ is also the rank of apparition of the prime $p$ for the number $\Delta_{n}= \pm \operatorname{Res}\left\{x^{n}-1, F(x)\right\}$ studied recently by D. H. Lehmer and others. (Annals of Mathematics, (2), vol. 34 (1933), pp. 461-479.)

$\ddagger$ In the case $k=2, \omega$ is the rank of apparition of the prime $p$ for the Lucas function $U_{n}$ associated with the polynomial $x^{2}-c_{1} x-c_{2}$ (D. H. Lehmer, Annals of Mathematics, (2), vol. 31 (1930), p. 422). In the general case, $\omega$ has been termed the restricted period of $F(x)$ modulo $p$ (R. D. Carmichael, Quarterly Journal of Mathematics, vol. 48 (1920), p. 354). 
2. Proof of the Theorem. We write as usual $a \mid b$ for $a$ divides $b$, and $(a, b)$ for the greatest common divisor of $a$ and $b$. Denote the roots of $F(x)=0$ in the finite field $\mathcal{F}$ by $\alpha, \alpha^{p}, \cdots, \alpha^{p^{k-1}}$. Then

$$
\text { norm } \alpha \equiv \alpha^{q}(p) \text {, }
$$

where $q=1+p+p^{2}+\cdots+p^{k-1}$.

As in the theorem, let $\omega$ denote the least positive value of $n$ such that $\alpha^{n}$ is congruent to a rational integer modulo $p$. Then every other such $n$ is readily seen to be divisible by $\omega$. In particular,

$$
\sigma=q / \omega=\left(p^{k}-1\right) /(\omega(p-1))
$$

is a rational integer, and

$$
\text { norm } \alpha \equiv M^{\sigma}(p)
$$

where $\alpha^{\omega} \equiv M(p),(1 \leqq M \leqq p-1)$.

Let $\lambda$ be the exponent to which $M$ belongs modulo $p, \theta$ the exponent to which norm $\alpha$ belongs modulo $p$, and $\tau$ the period of $\alpha$ in $\mathcal{F}$. Then

$$
\tau=\delta \theta \omega
$$

where $\delta=(\lambda, \sigma)$.

For since $\alpha^{\lambda \omega} \equiv M^{\lambda} \equiv 1(p), \tau \mid \lambda \omega$, and since $\alpha^{\tau}$ is congruent to a rational integer modulo $p, \omega \mid \tau$. Therefore, $\tau=\nu \omega$, where $\nu \mid \lambda$. Then $\alpha^{\tau}=\alpha^{\nu \omega} \equiv M^{\nu} \equiv 1(p)$, so that $\nu \mid \lambda$. Hence $\nu=\lambda, \tau=\lambda \omega$.

Now write $\lambda=\delta \lambda^{\prime}, \sigma=\delta \sigma^{\prime}$, where $(\lambda, \sigma)=\delta,\left(\lambda^{\prime}, \sigma^{\prime}\right)=1$. Then (norm $\alpha)^{\lambda^{\prime}} \equiv M^{\lambda^{\prime} \sigma}=M^{\lambda \sigma^{\prime}} \equiv 1(p)$, so that $\theta \mid \lambda^{\prime}$. Moreover, we have $M^{\theta \sigma} \equiv(\text { norm } \alpha)^{\theta} \equiv 1(p)$, so that $\lambda\left|\theta \sigma, \lambda^{\prime} \delta\right| \theta \delta \sigma^{\prime}, \lambda^{\prime} \mid \theta \sigma^{\prime}$, $\lambda^{\prime} \mid \theta$. Therefore $\lambda^{\prime}=\theta$ and $\lambda=\delta \lambda^{\prime}=\delta \theta, \tau=\lambda \omega=\delta \theta \omega$. Finally,

$$
(\theta, \sigma)|\delta|(k, p-1) \text {. }
$$

For since $\theta|\lambda,(\theta, \sigma)|(\lambda, \sigma)=\delta$, and since

$$
q=\left((p-1+1)^{k}-1\right) /(p-1) \equiv k(p-1),
$$

we have $(q, p-1)=(k, p-1)$. Therefore, since $\delta|\lambda| p-1$ and $\delta|\sigma| q, \delta \mid(q, p-1)$, it follows that $\delta \mid(k, p-1)$. Equations (1) and (2) give us our theorem.

3. Conclusion. To illustrate the theorem, consider the Fibo- 
nacci series $0,1,1,2,3,5,8,13, \cdots$ giving the values of the Lucas function $U_{n}$ associated with the polynomial $x^{2}-x-1$. This polynomial is irreducible modulo 13 , so that the period of the Fibonacci series modulo 13 gives the period of the mark $\alpha$ associated with $x^{2}-x-1$ in the finite field of order $13^{2}$. We have $\omega=7$, norm $\alpha=-1, \theta=2, k=2, \sigma=2, p-1=12$. Hence (2) becomes $(2,2)|\delta|(2,12)$, so that $\delta=2$. Hence the period is 28 , which is easily verified directly. It seems quite difficult to determine the exact value of $\delta$ in all cases. ${ }^{*}$

California Institute of Technology

\section{ON A PROBLEM OF KNASTER AND ZARANKIEWICZ $\dagger$}

BY J. H. ROBERTS

Knaster and Zarankiewicz have proposed the following problem: $\ddagger$ "Does every continuum $A$ contain a subcontinuum $B$ such that $A-B$ is connected?" Knaster has shown, $\S$ by an example in 3 -space, that the answer is in the negative. In the present paper an example is given of a plane continuum $M$ such that every non-degenerate proper subcontinuum of $M$ disconnects $M$.

The point sets considered in this paper all lie in a plane.

Definition of $F(C ; X, Y ; \epsilon)$. Let $C$ be any simple closed curve, $X$ and $Y$ distinct points of $C$, and $\epsilon$ any positive number. There exists a finite set of points $A_{1}, A_{2}, \cdots, A_{n},(n>2)$, such that (a) $A_{1}+A_{2}+\cdots+A_{n}$ contains $X+Y$, (b) $A_{1}, A_{2}, \cdots, A_{n}$ lie on $C$ in the order $A_{1} A_{2} \cdots A_{n} A_{1}$, and (c) $A_{i}$ and $A_{i+1}$ (subscripts are to be reduced modulo $n$ ) are the end points of an arc $t_{i}$ of diameter $<\epsilon$ which is a subset of $C$ not containing $A_{i+2}$. There exists a set of mutually exclusive arc segments $v_{1}, v_{2}, \cdots, v_{n}$ lying within $C$ such that $v_{i}+t_{i}$ is a simple closed curve $w_{i}$ of diameter $<\epsilon$. Let $J$ denote the simple closed curve

* See the discussion at the close of my paper, Transactions of this Society, vol. 33 (1931), p. 165.

$\dagger$ Presented to the Society, December 1, 1933.

$\ddagger$ Fundamenta Mathematicae, vol. 8 (1926), Problem 42, p. 376.

$\$$ B. Knaster, Sur un continu que tout sous-continu divise, Proceedings of the Polish Mathematical Congress, 1929, p. 59. 